Instructions for authors, subscriptions and further details:

\title{
Participación Política, Medios de Comunicación y Redes Sociales en los Adolescentes Catalanes
}

Sergio Villanueva Baselga ${ }^{1}$

Carlos Aguilar $^{1}$

Lydia Sánchez ${ }^{1}$

1) University of Barcelona, Spain

Date of publication: February $25^{\text {th }}, 2017$

Edition period: February 2017-June 2017

To cite this article: Villanueva Baselga, S., Aguilar, C., Sánchez, L. (2017).

Participación Política, Medios de Comunicación y Redes Sociales en los

Adolescentes Catalanes. International Journal of Sociology of Education, 6(1), 61-84. doi: 10.17583/rise.2017.2496

To link this article: http://dx.doi.org/10.17583/rise.2017.2496

PLEASE SCROLL DOWN FOR ARTICLE

The terms and conditions of use are related to the Open Journal System and to Creative Commons Attribution License (CC-BY) 


\section{Political Participation, Media and Social Networks Use by Catalan Adolescents}

Sergio Villanueva Baselga

University of Barcelona

\author{
Carlos Aguilar
}

University of Barcelona
Lydia Sánchez

University of Barcelona

(Received: 9 January 2017; Accepted: 26 January 2017; Published: 25 February 2017)

\section{Abstract}

The degree of political involvement of adolescents is an open question that crosses academic debate generation after generation. Currently, this issue is punctuated by the emergence of social networks and new media. This article examines the link between the use of media and social networks, and the interest for politics of a large sample of final year of secondary school Catalan students. The research concludes that the dynamics of political participation and discussion platforms used by teens do not differ too much from the older generations and, therefore, that social networks seem not to have affected the degree of political involvement of adolescents..

Keywords: political participation, civil engagement, social networks, media, adolescents 
RISE - International Journal of Sociology of Education Vol.6 No.1 Feburary 2017 pp. 61-84

\section{Participación Política, Medios de Comunicación y Redes Sociales en los Adolescentes Catalanes}

Sergio Villanueva Baselga

University of Barcelona
Carlos Aguilar

University of Barcelona
Lydia Sánchez

University of Barcelona

(Recibido: 9 Enero 2017; Aceptado: 26 Enero 2017; Publicado: 25 Febrero 2017)

\section{Resumen}

El grado de implicación política de los adolescentes es una cuestión abierta que atraviesa el debate académico generación tras generación. Actualmente, esta cuestión se ve salpicada por la irrupción de las redes sociales y los nuevos medios. El presente artículo estudia la vinculación entre el uso de medios, redes sociales y el interés por la política de una amplia muestra de estudiantes catalanes del último curso de Educación Secundaria Obligatoria. La investigación concluye que las dinámicas de participación política y las plataformas de debate de los adolescentes no difieren en exceso de las generaciones mayores y que, por tanto, las redes sociales no parecen haber afectado al grado de implicación política de los adolescentes.

Palabras clave: participación política, implicación cívica, redes sociales, medios de comunicación, adolescentes 


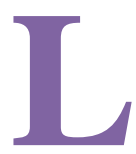

a generación Net es aquella que está compuesta por jóvenes que siempre han vivido rodeados de medios digitales y entornos virtuales y que, por lo tanto, poseen mayores conocimientos que sus padres y adultos que les rodean sobre nuevas tecnologías de la comunicación (Ballano et al, 2014). Brundidge \& Rice (2009) y Chadwick (2012) creen que Internet y los medios digitales han provocado dos tipos de influencias opuestas en lo que se refiere al grado de compromiso político de los jóvenes. Putman (2000) por su lado postula que Internet debilita el grado de compromiso y participación política, argumentando que se trata de un medio que distrae de aquellos temas que se suponen cruciales.

Con Internet ocurre lo que suele pasar cuando un nuevo medio de comunicación irrumpe en la sociedad. Una corriente de pensamiento enfatiza los posibles efectos negativos del nuevo medio, que reduciría el nivel de participación política. Por otro lado, otros pensadores subrayan lo contrario, llegando a afirmar que su influencia en la participación política sería positiva. Entre los primeros, Wojcieszak \& Mutz (2009) y Gaines \& Mondak (2009) sostienen la hipótesis de que Internet propicia que los ciudadanos formen redes sociales homogéneas, carentes de la variedad informativa necesaria para la formación política de los ciudadanos. Por el contrario, aquellos que ven en Internet un medio que facilita la participación política, se basan en su potencial democrático, su capacidad para llegar a una amplia audiencia, de asociación con otros individuos (Farrell, 2012), y de acceso a diferentes puntos de vista, trascendiendo las distancias físicas (Brundidge \& Rice, 2009).

Investigaciones recientes muestran la importancia que sigue teniendo la comunicación interpersonal en la decisión de voto de los ciudadanos. Las conversaciones en el seno de la familia, entre amigos, vecinos, siguen teniendo una influencia considerable en la elección del voto (Huckfeldt \& Sprague, 1995), aunque dichas conversaciones se suelan dar entre individuos que comparten un mismo tipo de visión de la realidad social (Mutz \& Martin, 2001). La idea es que del mismo modo que los espacios de opinión pública pueden articular el conocimiento político (Fishkin \& Luskin, 1999), e incidir en la opinión política (Gastil \& Dillard, 1999; Sturgis, Roberts, \& Allum, 2005), también lo hacen las conversaciones informales que mantienen los ciudadanos (Price, Cappella, \& Nir, 2002).

En este sentido, Boyd et al (2011) realizaron un estudio longitudinal que 


\section{Villanueva, Aguilar \& Sánchez-Participación política}

analizó información de 728 jóvenes de entre 13 y 16 años sobre sus perspectivas respecto al deber cívico, eficacia cívica, conexión social vecinal y participación cívica. El estudio confirma que una de las maneras en que el uso de las noticias que ofrecen los medios influye en la participación y deberes cívicos de los jóvenes es a través de la comunicación interpersonal con sus padres.

\section{Objetivos de la Investigación}

Frente al acceso clásico a la información a través de la prensa escrita y los informativos de las cadenas de televisión, el acceso de los adolescentes al contenido (de cualquier índole) está mayoritariamente mediado por los dispositivos de los que disponen. Según datos de la tercera ola del barómetro del EGM a fecha del estudio, para el grupo de edad comprendido entre $14 \mathrm{y}$ 19 años, la penetración de medios estimada en el periodo entre semana muestra un predominio de Internet $(90 \%)$, la televisión $(86 \%)$, la radio (60\%) y finalmente la prensa escrita con sólo un $22 \%$.

En esta línea, el objetivo principal del presente estudio es tratar de validar si los jóvenes de último año de enseñanza obligatoria utilizan las redes sociales como plataformas de información y discusión política, en detrimento de aquellos que utilizan los medios de comunicación clásicos para obtener esta información. Por ello es necesario profundizar y determinar si la universalización del acceso a la red ligado a la portabilidad de los dispositivos y las conexiones móviles, tiene alguna repercusión sobre la manera en la que los adolescentes se informan en su contexto sociopolítico. Más aun, es necesario determinar si la posibilidad de información y discusión política mediada por la red, está relacionada con el comportamiento político de los adolescentes.

Así, se hace necesario tratar de establecer si es mediante el uso de las redes sociales como los adolescentes obtienen sus fuentes de información política y finalmente cotejar si el paradigma de los nativos digitales es de aplicación en lo que respecta a las fuentes de acceso a la información de interés político. Por ello este artículo establece la relación entre tres variables: la utilización de los medios, el nivel de uso de las redes sociales y el nivel de interés político de los adolescentes. Los resultados analizados por separado y con las correlaciones establecidas entre ellos nos permiten 
elaborar una imagen de la relación existente entre intención de participación política, utilización de medios de comunicación y uso de las redes sociales. Además, se coteja si el uso de Internet como lugar de discusión política está relacionado con un mayor interés político, o si por el contrario, en la línea de lo ya referido por Huckfeldt \& Sprague (1995) es la discusión política entre conocidos y por canales tradicionales la que tiene un mayor nivel de influencia en el interés político de los jóvenes.

\section{Metodología}

La falta de consenso sobre si los medios digitales inciden en la participación política y, si lo hacen, qué tipo de efecto produce entre los jóvenes, pone sobre la mesa la necesidad de realizar estudios empíricos que ayuden a dilucidar este problema conceptual (Anduiza et al, 2009). Es en este contexto en el que este trabajo debe situarse. Presentamos un estudio empírico realizado sobre una muestra de 1709 estudiantes de cuarto curso de la ESO del territorio catalán, usando como instrumento de recogida de datos la encuesta detallada en Prats et al (2017) y Prats et al (2016). Mediante este estudio pretendemos averiguar hasta qué punto el nivel de uso de las redes sociales y las fuentes de información clásicas es homogéneo en el colectivo, y qué relación se establece entre la utilización de los medios y el nivel de implicación política de los jóvenes.

El grado de interés político en jóvenes y adolescentes se ha medido tradicionalmente no sólo por el nivel de participación de estos en partidos y compañas políticas - mismos criterios que sus pares adultos (Hooge and Wilkenfeld, 2008)-, sino también por su grado de implicación en actividades cívicas (Torney-Purta, 2002). En nuestro estudio, pese a que el grado de implicación en actividades cívicas también ha sido incluido en la encuesta, se toman como referencia las cuestiones que permiten incluir la intención declarada de interés político del conjunto de los encuestados, para poder contrastarla con la utilización que hacen de dispositivos y fuentes de información.

Con esta premisa, se utilizaron 3 variables (Tabla 1) para establecer el grado de interés político de los encuestados, cuyas respuestas estaban establecidas en una escala de Likert de 5 puntos ( $1=$ Totalmente en desacuerdo, $5=$ Totalmente de acuerdo): 
Q1. Me interesan las cuestiones políticas

Q2. Siempre intento aprender sobre cuestiones sociales y políticas actuales

Q3. Cuando tenga 18 años iré a votar de manera regular en las elecciones.

Tabla 1

Niveles de interés político

\begin{tabular}{lll}
\hline & $\mathrm{M}$ & $\mathrm{SD}$ \\
\hline Q1. Me interesan las cuestiones políticas & 2,78 & 1,280 \\
Q2. Siempre intento aprender sobre cuestiones & 2,90 & 1,264 \\
sociales y políticas actuales & & \\
$\begin{array}{l}\text { Q3. Cuando tenga 18 años iré a votar de manera } \\
\text { regular en las elecciones. }\end{array}$ & 3,45 & 1,267 \\
\hline
\end{tabular}

Las tres variables están además fuerte y positivamente correlacionadas, como sería de esperar (Tabla 2). Otorgando mayor nivel de intención de voto y mayor interés por informarse de asuntos políticos a aquellos que declaran un mayor grado de interés político. Por sexo hay ligeras variaciones siempre tendentes a mostrar un mayor interés político en los chicos, pero se pueden considerar significativas dada la desviación estándar obtenida.

Tabla 2

Correlación entre los elementos de interés político

\begin{tabular}{lll}
\hline & Q3. & Q2. \\
\hline Q1. & $0.505^{* *}$ & $0.784^{* *}$ \\
Q2. & $0.511^{* *}$ & 1 \\
\hline$\rho<0.01$ (2 colas) & & \\
\hline
\end{tabular}

Si bien una parte de la literatura sostiene la idea general de que los adolescentes presentan un alto grado de desafección política, esta idea no se ve reflejada en las intenciones de ejercer el voto de manera regular. Las dos opciones que son favorables rozan el 52\% (un resultado similar al de las votaciones municipales de 2015). Entre los 3 no desfavorables se llega al $79 \%$ de los adolescentes encuestados. 
Además, el desinterés por las cuestiones políticas es relativo, solo el 38,7 $\%$ se muestra en desacuerdo o muy en desacuerdo con laQ1 y tan solo 36,6 $\%$ se muestra en desacuerdo con la Q2. El resultado parece estar más de acuerdo con las conclusiones obtenidas por Henn et al. (2002) donde se cuestiona la idea general de que los jóvenes no tienen interés en la política. Tema aparte, fuera del alcance del estudio, es si este interés cristaliza posteriormente en una acción política, o se desvanece al no sentirse los jóvenes representados en el panorama político tradicional.

\section{Resultados}

\section{Utilización de Dispositivos para Conectarse a Internet}

Antes de explorar el uso de los medios como elementos determinantes en el nivel de interés político, nos preguntamos por el uso común que hacen los jóvenes de dispositivos. Los resultados del uso de dispositivos se midieron en una escala de Likert de 0 a 5 . Donde 0 es nunca, 1 es menos de una vez al mes, 2 menos de una vez a la semana, 3 menos de 1 vez al día, 1, entre 2 y 5 veces al día, 5 más de 5 veces al día

Los resultados muestran una clara supremacía de la utilización del teléfono móvil $(\mathrm{M}=4,24 \mathrm{SD}=1,42)$ frente al ordenador $(\mathrm{M}=3,54, \mathrm{SD}=1,08)$ o la Tablet $(\mathrm{M}=1,55, \mathrm{SD}=1,772)$, con ligeras diferencias en sexo siendo ligeramente superior el uso del ordenador (3,72 frente a 3,34) y la Tablet (1,6 frente a 1,51) por parte de los chicos, y de los teléfonos móviles en el caso de las chicas (4,32 frente a 4,18). La alta dispersión en el valor de la utilización de tabletas frente a la baja del uso de ordenadores muestran hasta qué punto la implantación de las tabletas no es aún uniforme en la población frente al ordenador o en menor medida al teléfono móvil como herramienta de acceso a Internet.

Para determinar el nivel de utilización de las redes sociales se les preguntó (de nuevo una escala de Likert de seis puntos) sobre la frecuencia con la que utilizaban las redes sociales, desde qué dispositivos, y preguntándose de cada una de ellas si se utiliza para seguir contactos o consultar contenidos, o se utiliza para crearlos, y en el caso de Twitter se distingue entre crearlos y /o retuitearlos. 
Tabla 3

Utilización de SNS según sexo.

\begin{tabular}{lllllll}
\hline & Chicos & & Chicas & \multicolumn{3}{c}{ Total } \\
\hline Actividad según red social & M & SD & M & SD & M & SD \\
\hline Facebook. Seguir contactos & 3,02 & 1,59 & 2,80 & 1,61 & 2,91 & 1,60 \\
Facebook. Crear contenidos & 2,30 & 1,57 & 2,65 & 1,56 & 2,47 & 1,58 \\
Tuenti. Seguir contactos & 0,33 & 1,01 & 0,31 & 0,91 & 0,32 & 0,97 \\
Tuenti. Crear contenidos & 0,28 & 0,93 & 0,29 & 0,87 & 0,29 & 0,91 \\
Twitter. Seguir contactos & 1,39 & 1,76 & 1,53 & 1,72 & 1,46 & 1,74 \\
Twitter. Retuitear & 1,29 & 1,73 & 1,70 & 1,91 & 1,49 & 1,83 \\
Twitter. Crear Tuits & 1,12 & 1,60 & 1,53 & 1,82 & 1,32 & 1,72 \\
Youtube. Mirar vídeos & 3,99 & 1,14 & 3,77 & 1,11 & 3,88 & 1,13 \\
Youtube. Subir vídeos & 0,72 & 1,27 & 0,31 & 0,89 & 0,52 & 1,12 \\
Instagram. Subir fotos & 1,19 & 1,54 & 2,10 & 1,63 & 1,63 & 1,65 \\
Instagram. Comentar fotos & 1,24 & 1,66 & 2,07 & 1,72 & 1,65 & 1,74 \\
Blogs. Leer blogs & 0,88 & 1,36 & 1,08 & 1,46 & 0,98 & 1,41 \\
Blogs. Hacer comentarios & & & & & & 1,01 \\
en blogs & 0,45 & 1,09 & 0,34 & 0,91 & 0,40 & 1,01 \\
Blogs. Mantener un blog & 0,38 & 1,00 & 0,38 & 1,04 & 0,38 & 1,03 \\
Forum. Leer fórums & 0,71 & 1,30 & 0,50 & 1,08 & 0,61 & 1,21 \\
Forum. Escribir a fórums & 0,34 & 0,99 & 0,12 & 0,56 & 0,24 & 0,84 \\
\hline
\end{tabular}

Los comportamientos de los adolescentes respecto al uso de las redes sociales muestran una alta penetración del uso de Youtube $(\mathrm{M}=3,88 \mathrm{SD}=$ $1,13)$ y Facebook $(\mathrm{M}=2,91 \mathrm{SD}=1,60)$ y en lo referente a la consulta de contenidos, seguidos por Instagram $(\mathrm{M}=1,63 \mathrm{SD}=1,65)$ y Twitter $(\mathrm{M}=1,46$, $\mathrm{SD}=1,74)$. Mientras en términos de creación de contenidos, es claramente Facebook la SNS más utilizada $(\mathrm{M}=2,47, \mathrm{SD}=1,57)$ seguida por Instagram $(\mathrm{M}=1,65, \mathrm{SD}=1,74)$ y Twitter $(\mathrm{m}=1,49, \mathrm{SD}=1,83)$. Los datos para Twitter e Instagram presentan una gran asimetría en términos de uso por sexo.

Visualizar videos en Youtube y seguir contactos en el Facebook son las ocupaciones mayoritarias en las redes sociales de los adolescentes encuestados. Existen sesgos de comportamientos asociados al sexo, en particular en lo referente a la creación de contenidos, siendo los chicos 
notablemente más activos en fórums, blogs y en especial en Youtube a la hora de generar contenidos, y la chicas en la utilización Twitter y muy especialmente de Instagram (Anexo1).

Los resultados muestran cómo los hábitos de uso de las diferentes redes sociales están íntimamente ligados al tipo de dispositivo. Establecidas las correlaciones entre el nivel de utilización de los dispositivos (ordenador, teléfono móvil o tableta) y el nivel de utilización de las redes sociales, se pueden distinguir claramente elementos de correlación significativos entre estas dos variables (tabla 4 ).

\section{Tabla 4}

Relación entre el uso de dispositivos y SNS

\begin{tabular}{llll}
\hline Uso de las SNS & Ordenador & Teléfono móvil & Tableta \\
\hline Facebook. Seguir contactos & $0,109 * *$ & $0,118^{* *}$ & $0,089^{* *}$ \\
Facebook. Crear contenidos & 0,037 & $0,170^{* *}$ & $0,126^{* *}$ \\
Tuenti. Seguir contactos & $0,058^{*}$ & 0,019 & $0,086^{* *}$ \\
Tuenti. Crear contenidos & $0,060^{*}$ & 0,024 & $0,098^{* *}$ \\
Twitter. Seguir contactos & $0,114 * *$ & $0,102 * *$ & $0,108^{* *}$ \\
Twitter. Retuitear & $0,095^{* *}$ & $0,141^{* *}$ & $0,093^{* *}$ \\
Twitter. Crear Tuits & $0,103 * *$ & $0,133^{* *}$ & $0,084^{* *}$ \\
Youtube. Mirar vídeos & $0,239^{* *}$ & $0,082^{* *}$ & $0,115^{* *}$ \\
Youtube. Subir vídeos & $0,190^{* *}$ & $-0,018$ & $0,091^{* *}$ \\
Instagram. Subir fotos & $-0,094^{* *}$ & $0,267 * *$ & $0,171^{* *}$ \\
Instagram. Comentar fotos & $-0,045$ & $0,257 * *$ & $0,200^{* *}$ \\
Blogs. Leer blogs & $0,125^{* *}$ & $-0,014$ & 0,038 \\
Blogs. Hacer comentarios en blogs & $0,093 * *$ & $-0,019$ & $0,061 *$ \\
Blogs. Mantener un blog & $0,093 * *$ & $-0,035$ & 0,036 \\
Forum. Leer fórums & $0,140^{* *}$ & $-0,075^{* *}$ & 0,042 \\
Fórums. Escribir en fórums & $0,137^{* *}$ & $-0,078^{* *}$ & 0,043 \\
\hline
\end{tabular}

$* * \rho<0,05 ; * \rho<0,1$

La utilización de Facebook está correlacionada con el uso de todos los tipos de dispositivos, pero lo que resulta notable es el hecho de que la 


\section{Villanueva, Aguilar \& Sánchez-Participación política}

participación activa en Facebook, (es decir que además de consultar su perfil, postean en él o en otros) está correlacionada con el uso de teléfonos inteligentes y tabletas, pero no con el uso del ordenador. Este comportamiento es análogo en Twitter y especialmente significativo en el caso de Instagram, donde se produce el mayor grado de correlación entre utilización de tabletas y teléfonos inteligentes con la utilización y el comentario de contenidos. Los resultados muestran que existe una correlación negativa entre el uso de Instagram y el del ordenador.

En contraposición a esta tendencia, existe una correlación entre el uso del ordenador y los niveles de uso y participación en fórums y blogs. Esta correlación se establece negativa al valorar la relación del uso de los teléfonos inteligentes y la participación en Blogs y Fórums.

Caso aparte es YouTube, en que las correlaciones son positivas para todo tipo de dispositivo si bien más acentuadas para ordenadores y tabletas, pero especialmente en lo referente a la participación. Este resultado es coherente con las necesidades de ancho de banda ligadas a la publicación de contenidos y a las limitaciones de tráfico establecidas por los operadores para las tarifas más habituales de telefonía móvil.

Establecida la dinámica de comportamiento de los jóvenes respecto a la utilización de dispositivos y redes sociales, hay que ver si estas conductas están relacionadas con las dinámicas de obtención de información, especialmente de información política.

\section{Fuentes de Información de los Adolescentes}

Cuando los adolescentes fueron preguntados sobre los medios y redes sociales que consumen para estar informados, se les presentaban cuatro posiciones ${ }^{1}$ en función del uso que se hacen de los medios para informarse de cuestiones políticas (tabla 5). 


\section{Tabla 5}

\begin{tabular}{lcc}
\multicolumn{3}{c}{ Utilización de fuentes de información } \\
\hline Medios y Redes sociales & Utilización \% & Utilización para informarse de temas políticos \% \\
\hline Diarios (n=1696) & 49,17 & 20,70 \\
Radio (n=1680) & 61,07 & 10,14 \\
Televisión (n=1681) & 94,81 & $\mathbf{3 9 , 3 3}$ \\
Tuenti (n=1664) & 23,62 & 2,71 \\
Facebook (n=1684) & 89,79 & 8,14 \\
Twitter (n=1675) & 53,24 & 9,20 \\
Instagram (n=166) & 62,95 & 2,06 \\
Blogs (n=1676) & 36,87 & 9,02 \\
Fórums (n=1672) & 29,01 & 7,96
\end{tabular}

El medio más utilizado a la hora de informarse (en términos generales) es la televisión, estando Facebook ligeramente por detrás. A continuación vemos que la utilización de la radio es equivalente al uso de Instagram, mientras que los diarios son utilizados como fuentes de información por menos del $50 \%$ de los adolescentes. Por tanto en términos generales, las redes sociales están al mismo nivel que las fuentes clásicas de información, pero no por encima de ellas. Los adolescentes han adoptado las redes sociales, como fuentes de información complementarias, pero no sustitutivas a la hora de conformar su información respecto al mundo que les rodea.

Sin embargo, a la hora de informarse de temas políticos, los medios que los adolescentes encuestados eligen son la televisión (39\%), los diarios (20\%), la radio (10\%), Twitter, los blogs y en sexto lugar está Facebook. $(8,14 \%)$. Los adolescentes siguen considerando que la televisión y los diarios son sus principales fuentes de información política, mientras que reservan las SNS para otros usos. Estos datos contradicen los obtenidos en estudios previos (Mitchell et al., 2015) que afirman que los adolescentes obtienen su información política a través de Facebook, mucho más que a través de los noticiarios tradiciones. 


\section{Villanueva, Aguilar \& Sánchez-Participación política}

Otra de las asunciones comunes al hablar de las conductas de los adolescentes es que no siguen las noticias en la televisión. Preguntados por sobre qué telenoticias ven de entre los presentados, podían escoger entre tres opciones (Nunca $=0$, Algunas veces $=1$, Regularmente=2). El 64,9\% de los encuestados afirman ver uno o más telediarios con regularidad, y sólo el $7,7 \%$ afirman no ver nunca ningún telediario. De nuevo, estos datos contrastan con buena parte de la literatura (tabla 6).

Tabla 6

Consumo de telenoticias por los adolescentes.

\begin{tabular}{lllll}
\hline Telediarios & Alumnos & \multicolumn{3}{l}{ Padres } \\
\hline \multirow{2}{*}{ Telenoticias TV3 } & $\mathrm{M}$ & $\mathrm{SD}$ & $\mathrm{M}$ & $\mathrm{SD}$ \\
8 al día & 0,71 & 0,81 & 0,99 & 0,89 \\
Telediario TV1 & 0,16 & 0,46 & 0,40 & 0,70 \\
Informativos Tele5 & 0,53 & 0,70 & 0,86 & 0,83 \\
Antena 3 Noticias & 0,70 & 0,79 & 0,98 & 0,85 \\
La Sexta Noticias & 0,98 & 0,80 & 1,22 & 0,80 \\
La 2 Noticias & 0,51 & 0,68 & 0,84 & 0,82 \\
Noticias 4 & 0,15 & 0,42 & 0,35 & 0,63 \\
Noticias Locales & 0,77 & 0,80 & 0,92 & 0,80 \\
Noticias Extranjero & 0,32 & 0,58 & 0,46 & 0,69 \\
\hline
\end{tabular}

La diferencia por sexos es mínima (tabla 7), si bien pueden apreciarse diferencias significativas en los telediarios de Telecinco y A3, donde las chicas tienen una presencia mucha más significativa. Las causas de estas diferencias, aunque están fuera del alcance de este trabajo, pueden deberse a un efecto de arrastre de la programación previa de las cadenas (Boemer, 1987). 


\section{Tabla 7}

Relación entre el consumo de telenoticias y sexo

\begin{tabular}{lllll}
\hline & Chicos & \multicolumn{3}{l}{ Chicas } \\
\hline Telenoticias TV3 & M & SD & M & SD \\
8 al día & 0,75 & 0,82 & 0,67 & 0,80 \\
Telediario TV1 & 0,18 & 0,47 & 0,14 & 0,44 \\
Informativos Tele5 & 0,51 & 0,69 & 0,54 & 0,70 \\
Antena 3 Noticias & 0,60 & 0,75 & 0,81 & 0,81 \\
La Sexta Noticias & 0,91 & 0,79 & 1,04 & 0,80 \\
La 2 Noticias & 0,53 & 0,69 & 0,48 & 0,67 \\
Noticias 4 & 0,15 & 0,42 & 0,15 & 0,41 \\
Noticias Locales & 0,88 & 0,83 & 0,65 & 0,75 \\
Noticias Extranjero & 0,32 & 0,59 & 0,31 & 0,57 \\
\hline
\end{tabular}

En esta línea, las correlaciones entre el telediario que se acostumbra a ver y el nivel de interés político muestran una distinción entre dos grupos de telenoticias: aquellos correlacionados con el interés político y aquellos que no lo están, o incluso que lo están negativamente (tabla 8).

Tabla 8

Correlación entre visionado de Telenoticias e interés político

\begin{tabular}{lccc}
\hline & Q1 & Q2 & Q3 \\
\hline Telenoticias TV3 &, $275^{* *}$ &, $259^{* *}$ &, $267^{* *}$ \\
8 al día &, $155^{* *}$ &, $122^{* *}$ &, $104^{* *}$ \\
Telediario TV1 &, $060^{* *}$ &, $063^{* *}$ &, 031 \\
Informativos Tele5 &,- 028 &,- 002 &,- 031 \\
Antena 3 Noticias &,- 033 &,- 003 &,$- 051^{*}$ \\
La Sexta Noticias &, $063^{* *}$ &, $084^{* *}$ &, 012 \\
La 2 Noticias &,- 010 &,- 016 &,- 047 \\
Noticias 4 &,- 014 &, 030 &, 002 \\
Noticias Locales &, 039 &, 033 &, 035 \\
Noticias Extranjero &, 031 &, 016 &,$- 057^{*}$ \\
\hline$* * \rho<0,05 ; * \rho<0,1$ & \multicolumn{3}{c}{} \\
\hline
\end{tabular}




\section{Villanueva, Aguilar \& Sánchez-Participación política}

Los adolescentes que ven con mayor asiduidad los telenoticias de Televisión de Catalunya, 8 TV, TVE y La Sexta, son aquellos que tienen un mayor interés político. Si bien sólo se establece una correlación positiva entre el visionado de telenoticias y el interés de ejercer el derecho al voto. Este factor puede relacionarse con el mayor consumo de telenoticias en lengua catalana de aquellos colectivos más próximos a posiciones nacionalistas, mucho más movilizados en su intención de voto y consumidores habituales de noticias en esa lengua. Estos resultados están en consonancia con los obtenidos por Muñoz y Tormos (2012), que mostraron la relación existente entre los consumidores de Televisión de Catalunya y el mayor interés político, en particular una mayor tendencia hacia posiciones independentistas.

En paralelo, hay un grupo de noticiarios que se relacionan negativamente (si bien no de manera significativa) con el interés político. Especialmente relevante es el hecho de que el más visto de los telenoticias, Antena 3 noticias, sea el único de entre los telediarios nacionales correlacionado negativamente de manera significativa, con la intención de voto declarada. A la luz de estos datos, podemos afirmar que los sujetos con mayor interés político, eligen unos ciertos canales de televisión para informarse, al igual que eligen unos determinados medios u otros, y que los datos mostrados apuntan a una preocupante relación con la falta de interés político e incluso de interés de participación activa en las elecciones de los adolescentes.

Además, respecto a los medios utilizados para obtener información resulta especialmente significativa la correlación que se establece entre el nivel de interés político declarado por los adolescentes y la utilización de los medios como fuente de información política. A excepción de Tuenti e Instagram, la utilización de los medios de información se correlaciona, como es natural, con el nivel de interés político (tabla 9). 
Tabla 9

Utilización de medios para informarse

\begin{tabular}{lccc}
\hline & Q1 & Q2 & Q3 \\
\hline DIARIOS &, $198^{* * *}$ &, $211^{* * *}$ &, $178^{* *}$ \\
RADIO &, $114^{* *}$ &, $109^{* *}$ &, $150^{* *}$ \\
TELEVISIO &, $094^{* *}$ &, $114^{* *}$ &, $087^{* *}$ \\
TUENTI &,$- 069^{* *}$ &,$- 061^{* *}$ &,- 028 \\
FACEBOOK &, $092^{* *}$ &, $079^{* *}$ &, $058^{*}$ \\
TWITTER &, $078^{* *}$ &, $095^{* *}$ &, $072^{* *}$ \\
INSTAGRAM &, 025 &, 015 &, $085^{* *}$ \\
BLOCS &, 043 &, $066^{* *}$ &, 016 \\
FORUMS &, $074^{* *}$ &, $083^{* *}$ &, 019 \\
WHATSAPP &, $066^{* *}$ &, 047 &, $081^{* *}$ \\
\hline$* * \rho<0,05 ;{ }^{*} \rho<0,1$ & \multicolumn{3}{|}{} \\
\hline
\end{tabular}

Lo que resulta relevante, por tanto, es que son los adolescentes que utilizan los medios clásicos (diarios, radio y televisión) los que demuestran un mayor interés político.

Existen diversas discusiones que dominan la literatura sobre el nivel de participación política de los adolescentes. Por un lado la discusión sobre si los nuevos medios favorecen la participación política (De Zuñiga, 2009), y una segunda ligada al uso de las redes sociales como fuente principal de información política (Yoo y Gil de Zúñiga, 2014). Todo ello dentro de una discusión general sobre la falta de afección política de los jóvenes y adolescentes.

Los datos aportados en este trabajo muestran cómo entre los adolescentes catalanes los medios convencionales son sus fuentes de información política principales, si bien el uso de Facebook, Twitter y fórums, se correlaciona igualmente con un mayor interés político. Una conclusión innegable navega por encima de las dos discusiones, el mayor uso de las fuentes de información se corresponde con una mayor intención de ejercer el derecho al voto. Es decir, parece ser una norma general que el deseo de estar informado y el deseo de expresar la opinión política se ve reflejado en términos de ejercer el derecho al voto. 


\section{Villanueva, Aguilar \& Sánchez-Participación política}

Tabla 10

Uso de las redes sociales e interés político

\begin{tabular}{llll}
\hline Tipo de utilización de las redes & $\mathrm{Q} 1$ & $\mathrm{Q} 2$ & $\mathrm{Q} 3$ \\
\hline Facebook. Seguir contactos &,- 017 &,- 022 &,- 027 \\
Facebook. Crear contenidos &,$- 079^{* *}$ &,$- 091^{* *}$ &,$- 050^{*}$ \\
Twitter. Seguir contactos &, $064^{* *}$ &, $070^{* *}$ &, $056^{*}$ \\
Twitter. Retuitear &, $071^{* *}$ &, $086^{* *}$ &, $061^{*}$ \\
Twitter. Crear Tuits &, 048 &, $050^{*}$ &, 040 \\
Youtube. Mirar vídeos &,- 010 &,- 027 &,- 031 \\
Youtube. Subir vídeos &,- 002 &,- 033 &,- 040 \\
Instagram. Subir fotos &,- 042 &,$- 053^{*}$ &, 020 \\
Instagram. Comentar fotos &,- 020 &,- 037 &, $061^{*}$ \\
Blogs. Leer blogs &, $107^{* *}$ &, $110^{* *}$ &, 030 \\
Blogs. Hacer comentarios en blogs &, 034 &, 046 &,- 013 \\
Blogs. Mantener un blog &,- 027 &,- 030 &,- 037 \\
Forum. Leer Fórums &, $130^{* *}$ &, $134^{* *}$ &, $062^{*}$ \\
Forums. Escribir a fórums &, $081^{* *}$ &, $074^{* *}$ &, 018 \\
\hline
\end{tabular}

$* * \rho<0,05 ; * \rho<0,1$

Sin embargo, debe matizarse la relación entre el uso de las redes sociales y el interés político de los adolescentes. Mientras que se declara que Facebook es una fuente de información política, los datos muestran que aquellos adolescentes que hacen un uso más intensivo de esta red social, presentan una correlación negativa con el interés político, incluso con la intención de ejercer el voto. Por el contrario, los usuarios de fórums, blogs, y Twitter, pese a ser minoritarios, son los usuarios que muestran un mayor nivel de interés político, unos resultados coherentes con los presentados por estudios anteriores.

La novedad presentada en este estudio es la constatación de que el uso de Facebook, Youtube e Instagram, no está o está negativamente correlacionado con el interés político de los adolescentes. Este resultado viene a aportar luz, con datos empíricos, a la discusión sobre el uso que los adolescentes hacen de sus redes sociales, pudiendo afirmarse, que el uso intensivo que hacen de YTB y Facebook no repercute en un mayor interés 
por cuestiones políticas ni en una mayor intención a ejercer su derecho a voto. Los resultados confirman los comportamientos observados en el conjunto de la población que relaciona el uso de Twitter como red de información política por excelencia, así como la continuación del uso de blogs y fórums como fuente de información y discusión política.

¿Supone la Utilización de las Redes Sociales una Nueva Ventana de Discusión Política dado que Facilitan la Discusión entre Personas de Opiniones Políticas Confrontadas?

Este apartado explora la posibilidad de que la discusión política online suponga una diferencia respecto a la discusión política cara a cara, fomentado esta nueva posibilidad la discusión con personas de ideología diferente. En última instancia, se busca constatar o negar que aquellos que debaten activamente por internet, poseen un mayor interés político que los que no lo hacen.

Los datos reflejados en la tabla 11 nos indican que el nivel de discusión política de los adolescentes es significativamente inferior por Internet que en persona, dato que, de nuevo, esclarece el debate sobre el papel que la discusión política por Internet juega entre los adolescentes. 


\section{Villanueva, Aguilar \& Sánchez-Participación política}

Tabla 11

Nivel de discusión política presencial y por Internet

\begin{tabular}{lcc}
\hline & M & SD \\
\hline $\begin{array}{l}\text { ¿Con qué frecuencia hablas de política con los amigos? } \\
\text { ¿Con qué frecuencia hablas personalmente de temas políticos con }\end{array}$ & 2,25 & 1,11 \\
$\begin{array}{l}\text { las personas que tienen ideas muy diferentes de las tuyas? } \\
\begin{array}{l}\text { Cuando estás presente en una conversación cara a cara sobre } \\
\text { política y se expresan opiniones con las que no estás de acuerdo, }\end{array}\end{array}$ & 2,86 & 1,32 \\
$\begin{array}{l}\text { ¿con qué frecuencia participas en el debate? } \\
\text { ¿Con qué frecuencia hablas de política por Internet? }\end{array}$ & 1,46 & 0,84 \\
$\begin{array}{l}\text { En tus relaciones por Internet, con qué frecuencia hablas de temas } \\
\text { políticos con las personas que tienen ideas muy diferentes de las } \\
\text { tuyas? }\end{array}$ & 1,48 & 0,88 \\
$\begin{array}{l}\text { Cuando ves una conversación en Internet sobre política, y se } \\
\text { expresan opiniones con las que no estás de acuerdo, ¿con qué } \\
\text { frecuencia participas en el debate? }\end{array}$ & 1,65 & 1,08 \\
\hline
\end{tabular}

Respecto al lugar donde tiene lugar la discusión política y el nivel de interés político de los adolescentes los resultados se presentan en la tabla 12. 
Tabla 12

Lugar de discusión política y nivel de implicación política

\begin{tabular}{|c|c|c|c|}
\hline Lugar de discusión política: cara a cara & Q1 & Q2 & Q3 \\
\hline ¿Con qué frecuencia hablas de política cara a cara? &, $553^{* *}$ &, $493^{* *}$ &, $373^{* *}$ \\
\hline $\begin{array}{l}\text { ¿Con qué frecuencia hablas personalmente de temas políticos } \\
\text { con las personas que tienen ideas muy diferentes de las tuyas? }\end{array}$ &, $504^{* *}$ &, $469^{* *}$ &, $325^{* *}$ \\
\hline $\begin{array}{l}\text { Cuando estás presente en una conversación cara a cara sobre } \\
\text { política y se expresan opiniones con las que no estás de } \\
\text { acuerdo, ¿con qué frecuencia participas en el debate? }\end{array}$ &, $505^{* *}$ &, $467^{* *}$ &, $390^{* *}$ \\
\hline Lugar de discusión política: Internet & Q1 & Q2 & Q3 \\
\hline ¿Con qué frecuencia hablas de política por Internet? & $325^{* *}$ & $311^{* *}$ &, $188^{\text {** }}$ \\
\hline $\begin{array}{l}\text { En tus relaciones por Internet, ¿con qué frecuencia hablas de } \\
\text { temas políticos con las personas que tienen ideas muy }\end{array}$ & & & \\
\hline diferentes de las tuyas? &, $270 * *$ &, $282^{* *}$ &, $196^{* *}$ \\
\hline $\begin{array}{l}\text { Cuando ves una conversación en Internet sobre política, y se } \\
\text { expresan opiniones con las que no estás de acuerdo, ¿con qué }\end{array}$ & & & \\
\hline $\begin{array}{l}\text { frecuencia participas en el debate? } \\
* * \rho<0,05 ; * \rho<0,1\end{array}$ &, 29 &, 2 &, 215 \\
\hline
\end{tabular}

Parece, por tanto, que podemos afirmar que aquellos que habitualmente expresan sus opiniones sobre política cara a cara son los que tienen un mayor nivel grado de interés político. En este sentido, nuestro estudio contradice las conclusiones extraídas por estudios previos que mostraron que la exposición a debates llevados a cabo en Internet aumentaba el interés por la política.

\section{Conclusiones}

Los datos obtenidos muestran cómo los adolescentes realizan un uso intensivo de Internet y las redes sociales, y cómo este uso está ligado a los dispositivos utilizados, y que en algunos casos como el de Instagram, pueden observarse diferencias significativas en términos de género.

Pese a que buena parte de la bibliografía constata que el uso intensivo que hacen los adolescentes de Internet, y que se constata en este estudio, es importante para mejorar la participación online, los resultados obtenidos indican que esta utilización no refleja la manera en que Internet actúa como 


\section{Villanueva, Aguilar \& Sánchez-Participación política}

fuente de información política.

Como Ballano et al (2014) también mostraron a partir de un estudio cualitativo basado en 30 grupos focales, las nociones de nativos e inmigrantes digitales no son válidas en tanto en cuanto la capacidad de manejo de las ICT. Es decir, a pesar de que los miembros de la generación Net hayan convivido con las tecnologías de la información y la comunicación desde su nacimiento, poseen una motivación menor ante el aprendizaje de su manejo que las generaciones anteriores. Por ello, tal y como demuestra nuestro estudio, los patrones de uso de estas tecnologías para obtener información no difieren entre los estudiantes de secundaria y sus progenitores. Especialmente significativo resulta el hecho de que para informarse sobre política siguen recurriendo a las mismas fuentes de información (diarios, radio y televisión), pese a ser usuarios intensivos de las redes sociales.

Los resultados de este trabajo sí que permiten profundizar en las conclusiones de Yoo y Gil de Zúñiga (2014), en las que se afirmaba que Blogs, Twitter y Facebook parecen tener diferentes cualidades como medios de comunicación. Y si bien el uso de ellos como fuente de información política por parte de los adolescentes se correlaciona con el grado de interés político (Tabla 9), el análisis del uso que hacen de los mismos nos permite afirmar que en conjunto, tan solo los usos de blogs, fórums y Twitter, se corresponden realmente con un mayor nivel de interés político. Esto plantea importantes reflexiones de cara a futuros trabajos. Los adolescentes parecen tender a afirmar que entre los usos que hacen del conjunto de las redes sociales, está incluido el de su utilización como fuentes la información política, cuando el análisis detallado de sus usos, dista mucho de sostener dichas afirmaciones (Tabla 10), llegando al extremo de establecer correlaciones negativas entre la utilización de algunas redes sociales, Facebook principalmente, y el nivel de interés político de los adolescentes.

Sigue siendo el uso de los telenoticias el medio preferido por los adolescentes para informarse de temas políticos. Profundizando en las cadenas elegidas para realizarlo, se observa que el nivel de consumo es proporcional a las audiencias medias de los mismos. Sin embargo, existe una clara asociación entre el consumo de un subgrupo de ellos y el nivel de interés político de los adolescentes. Esto muestra cómo los adolescentes son activos en su interés político en lo que a la selección de fuentes de 
información se refiere, lo que nos lleva a cuestionar una parte de la literatura, que asocia a los jóvenes con un alto nivel de apatía respecto a las cuestiones políticas.

\section{Agradecimientos}

Este artículo se ha llevado a cabo dentro de los siguientes proyectos: grupo de investigación consolidado DHIGECS (Didáctica de la Historia, la Geografía y otras Ciencias Sociales), financiado por la Generalidad de Catalunya (2014SGR-955); proyecto Recercaixa 2012 «Educación cívica en les aulas interculturales: análisis de las representaciones e ideas sociales del alumnado y propuestas de acción educativa» (2012ACUP00185), financiado por la Obra Social "la Caixa"; proyecto «Desarrollo de la formación sociopolítica para una ciudadanía democrática: diseño e implementación de materiales didácticos en Ciencias Sociales» (EDU2015-65621-C3-3-R), financiado por el Ministerio de Economía y Competitividad del Gobierno de España.

\section{Referencias}

Anduiza, E., Cantijoch, M. y Gallego, A. (2009). Political participation and the Internet. Information, Communication and Society.12(6), 860 878. doi: 10.1080/13691180802282720

Anduiza, E., Cristancho, C., \& Cantijoch, M. (2012). La exposición a información política a través de internet. Arbor, 188(756), 673-688. doi: 10.3989/arbor.2012.756n4004

Ballano, S., Uribe, A.C. \& Munté-Ramos, R-A. (2014). Young users and the digital divide: readers, participants or creators on Internet?

Communication \& Society, 27(4), 147-155.

Boemer, M. L. (1987). Correlating lead-in show ratings with local television news ratings. Journal of Broadcasting \& Electronic Media, 31(1), 89-94. doi:10.1080/08838158709386647

Boyd, M. J., Zaff, J. F., Phelps, E., Weiner, M. B. y Lerner, R. M. (2011). The relationship between adolescents' news media use and civic engagement: The indirect effect of interpersonal communication with 
82 Villanueva, Aguilar \& Sánchez-Participación política

parents. Journal of Adolescence 34. doi:

10.1016/j.adolescence.2011.07.004

Brundidge, J., Rice, R. E. (2009). Political engagement online: Do the information rich get richer and the like-minded more similar. In A. Chadwick (Ed.), Routledge handbook of Internet politics (pp.144156). London, New York: Routledge.

Chadwick, A. (2012). Recent shifts in the relationship between the Internet and democratic engagement in Britain and the United States: granularity, informational exuberance and political learning. In Anduiza, E., Jensen, M. \& Jorba, L. (Eds.), Digital media and political engagement worldwide: a comparative study (pp.39-52). Cambridge: Cambridge University Press.

De la Fuente, J. R. (2010). Ciberpolítica, redes sociales y nuevas movilizaciones en España: el impacto digital en los procesos de deliberación y participación ciudadana. Mediaciones sociales, (7), 143-164.

De Zúñiga, H. G., Puig-i-Abril, E., \& Rojas, H. (2009). Weblogs, traditional sources online and political participation: An assessment of how the Internet is changing the political environment. New media \& society, 11(4), 553-574. doi: 10.1177/1461444809102960

Delli Carpini, M. X., Cook, F. L., \& Jacobs, L. R. (2004). Public deliberation, discursive participation, and citizen engagement: A review of the empirical literature. Annual Review of Political Science, 7, 315-344. doi: 10.1146/annurev.polisci.7.121003.091630

Farrell, H. (2012). The consequences of the Internet for politics. Political Science, 15(1), 35-52. doi: 10.1146/annurev-polisci-030810-110815

Fishkin, J. S., \& Luskin, R. C. (1999). Bringing deliberation to the democratic dialogue. In M. McCombs \& A. Reynolds (Eds.), The poll with a human face: The National Issues Convention experiment in political communication (pp. 3-38). Mahwah, NJ: Erlbaum.

Gaines, B. J., Mondak, J. J. (2009). Typing together? Clustering of ideological types in online social networks. Journal of Information Technology \& Politics, 6(3-4), 216-231. doi:

10.1080/19331680903031531

Henn, M., Weinstein, M., \& Wring, D. (2002). A generation apart? Youth and political participation in Britain. The British Journal of Politics 
and International Relations, 4(2), 167-192. doi: 10.1111/1467856X.t01-1-00001

Hooge, M and Wilkenfeld, B (2008) The Stability of Political Attitudes and Behaviors across Adolescence and Early Adulthood: A Comparison of Survey Data on Adolescents and Young Adults in Eight Countries. Journal of Youth and Adolescence. 37(2): 155-167 doi: 10.1007/s10964-007-9199-X

Huckfeldt, R., \& Sprague, J. (1995). Citizens, politics, and social communication: Information and influence in an election campaign. Cambridge, England: Cambridge University Press.

Gastil, J., \& Dillard, J. P. (1999). Increasing political sophistication through public deliberation. Political Communication, 16, 3-23.

Mitchell, A., Gottfried, J. and Matsa K.E. (2015) Milennians and Polítical News, Pew Research Center Analysis. Available in: http://www.journalism.org/2015/06/01 /millennials-political-news/

Moy, P., Gastil, J. (2006) Political Communication Predicting Deliberative Conversation: The Impact of Discussion Networks, Media Use, and Political Cognitions. Political Communication, 23, 443-460,

Muñoz, J., \& Tormos, R. (2012). Identitat o càlculs instrumentals? Anàlisi dels factors explicatius del suport a la independència. Barcelona: Centre d'Estudis d'Opinió.

Mutz, D. C., \& Martin, P. S. (2001). Facilitating communication across lines of political difference: The role of mass media. American Political Science Review, 95, 97-114.

Prats, J., Salazar-Jiménez, R., \& Molina-Neira, J. (2016). Implicaciones metodológicas del respeto al principio de autonomía en la investigación social. Andamios: revista de investigación social, 31(6), 129-154.

Prats-Cuevas, J., Molina-Neira, J., Ruiz-Bueno, A., Molina-Luque, F. (2017). Análisis de las Representaciones e Ideas Sociales del Alumnado en Educación para la Ciudadanía Democrática: Ejemplo de Mixed-methology desde y para la Investigación Transdisciplinar. International Journal of Sociology of Education, 6(1), 1-25. doi: 10.17583/rise.2017.2479 
84 Villanueva, Aguilar \& Sánchez-Participación política

Price, V., Cappella, J., \& Nir, L. (2002). Does disagreement contribute to more deliberative opinion? Political Communication, 19, 95-112. doi: 10.1080/105846002317246506

Putnam, R. D. (2000). Bowling alone: The collapse and revival of American community. New York: Touchstone.

Sturgis, P., Roberts, C., \& Allum, N. (2005). A different take on the deliberative poll: Information, deliberation, and attitude constraint. Public Opinion Quarterly, 69, 30-65. doi: 10.1093/poq/nfi005

Torney-Purta, J (2002) The School's Role in Developing Civic Engagement: A Study of Adolescents in Twenty-Eight Countries. Applied Developmental Science, 6(4), 203-212. doi: 10.1207/S1532480XADS0604_7

Wojcieszak, M. E., Mutz, D. C. (2009). Online groups and political discourse: do online discussion spaces facilitate exposure to political disagreement? Journal of Communication, 59(1), 40-56. doi: 10.1111/j.1460-2466.2008.01403.x

Yoo, S.W. \& Gil de Zúñiga, H. (2014). Connecting blog, Twitter and Facebook use with gaps in knowledge and participation. Communication \& Society, 27(4), 33-48

Sergio Villanueva Baselga is Professor at the University of Barcelona Spain.

Carlos Aguilar is Professor at the University of Barcelona Spain. Lydia Sánchez is Professor at the University of Barcelona Spain. Contact Address: sergio.villanueva@ub.edu 